\section{Encefalopatía subaguda en una paciente con policondritis recidivante. Caso clínico}

\author{
CONSTANZA VERA ${ }^{1}$, JOSÉ MANUEL MATAMALA ${ }^{1,2,3,4}$, \\ WALTER FEUERHAKE ${ }^{1}$, OSCAR NEIRA ${ }^{4}$, \\ AARON VIDAL ${ }^{5}$, JOSÉ LUIS CASTILLO
}

\section{Subacute encephalopathy associated with relapsing polychondritis. Report of one case}

Relapsing polychondritis $(R P)$ is a rare multisystemic autoimmune disorder characterized by the inflammation and destruction of cartilages, with preference for auricular, nasal and laryngotracheal cartilages. RP may also affect proteoglycan-rich structures, such as, blood vessels, eyes, kidneys, and heart. The central nervous system (CNS) is involved in less than 3\% of patients. We report a 32-year-old female with $R P$ associated with a progressive subacute encephalopathy characterized by behavioral disturbances, auditory and visual hallucinations. The EEG showed generalized slow activity and a mononuclear pleocytosis with increased protein was found in the cerebrospinal fluid. The brain magnetic resonance imaging showed multiple supra and infratentorial nodular inflammatory lesions. After initiating treatment with corticosteroids and cyclophosphamide, a significant improvement in chondritis and neurological status was observed.

(Rev Med Chile 2021; 149: 1085-1089)

Key words: Brain Diseases; Leukocytosis; Polychondritis, Relapsing.
'Departamento de Ciencias

Neurológicas Oriente, Facultad de Medicina, Universidad de Chile.

Santiago, Chile.

2Laboratorio de Neurología y Neurofisiología Traslacional, Centro de Investigación Clínica Avanzada (CICA) Oriente, Facultad de Medicina, Universidad de Chile. Santiago, Chile. ${ }^{3}$ Departamento de Neurociencia,

Facultad de Medicina, Universidad de Chile. Santiago, Chile.

${ }^{4}$ Instituto Milenio de Neurociencia Biomédica (BNI), Facultad de Medicina, Universidad de Chile. Santiago, Chile. ${ }^{5}$ Sección de Reumatología, Hospital del Salvador, Universidad de Chile.

Santiago, Chile.

${ }^{6}$ Servicio de Neurorradiología, Instituto de Neurocirugía Dr. Asenjo (INCA).

Santiago, Chile.

Trabajo no recibió financiamiento. Los autores declaran no tener conflictos de interés.

Recibido el 15 de mayo de 2020 , aceptado el 4 de agosto de 2021 .

Correspondencia a:

Dr. José Luis Castillo

José Manuel Infante 553, Providencia, Santiago, Chile.

jlcastillo57@gmail.com
L a policondritis recidivante $(\mathrm{PR})$ es un trastorno autoinmune multisistémico poco frecuente, descrito por primera vez en 1923 por Jakasch Watenhorst como una policondropatía degenerativa. La incidencia anual es de 3,5 casos por cada millón de habitantes, afectando por igual a hombres y mujeres. La enfermedad se caracteriza por la inflamación y destrucción, recurrente y progresiva de estructuras cartilaginosas (cartílago hialino, elástico y fibroso), principalmente auricular, nasal y laringotraqueal. Puede afectar también órganos ricos en proteoglicanos como ojos, corazón, riñón y vasos sanguíneos. La presencia de anticuerpos contra colágeno II, IX y XI puede ser identificada entre $1 / 5$ a $1 / 3$ de los pacientes. La enfermedad puede coexistir con otras patologías autoinmunes, como lupus eritematoso sistémico, artritis reumatoide y esclerosis sistémica, hasta en
35\% de las veces. Según McAdam¹ ${ }^{1}$ el diagnóstico se realiza con el hallazgo de tres características clínicas como: condritis auricular o nasal, poliartritis inflamatoria no erosiva, inflamación ocular, condritis del tracto respiratorio y daño cocleo-vestibular. Damiani y Levine ${ }^{2}$ proponen luego que el diagnóstico puede establecerse con tres de los criterios clínicos señalados, un criterio clínico más estudio histológico sugerente de condritis o bien dos o más características clínicas asociadas a la presencia de condritis en dos o más áreas anatómicamente separadas, y respuesta terapéutica tras la administración de esteroides o dapsona $^{1,2}$. El compromiso del sistema nervioso central (SNC) se describe solo 3\% de casos de $\mathrm{PR}$, cifra que ha sido obtenida del reporte de $\operatorname{casos}_{\text {clínicos }}{ }^{3-6}$. Se presume que el compromiso neurológico ocurre como resultado de fenómenos 
vasculíticos. Reportes post mortem han revelado infiltración perivascular de linfocitos $\mathrm{T}$ a nivel de la sustancia gris y sustancia blanca, con inflamación y destrucción de la mielina ${ }^{7,8}$.

\section{Caso clínico}

Se reporta el caso de una mujer de 32 años, $\sin$ antecedentes mórbidos. Consulta por cuadro progresivo de tres meses de evolución caracterizado por cefalea, confusión, bradipsiquia, cambios conductuales y alucinaciones tanto visuales como auditivas. Desde dos meses antes presentaba lesiones en cuero cabelludo, tipo alopecia cicatricial, con histología compatible con lupus cutáneo, y enrojecimiento intermitente de los pabellones auriculares. La determinación de anticuerpos antinucleares (ANA) fue negativa. Al ingreso al servicio de Neurología del Hospital del Salvador la paciente se encontró vigil, desorientada en tiempo y espacio, altamente distráctil, presentando dificultad para seguir órdenes complejas y enlentecimiento de la velocidad de respuesta sin alteraciones del lenguaje, apraxias ni agnosias; examen de pares craneanos sin hallazgos patológicos, fondo de ojo sin papiledema; al examen motor solo destacó hiperreflexia generalizada sin otros signos piramidales. Resto del examen dentro de rangos fisiológicos. Al examen general se objetiva leve aumento de volumen y eritema asimétrico de ambos pabellones auriculares, asociado a aumento de temperatura local, sin deformidad del pabellón auricular (Figura 1), además de las lesiones en cuero cabelludo previamente descritas. La evaluación neuropsicológica destaca test de mini-mental de 24/30 puntos, test de MOCA (Montreal Cognitive Assessment) de 19/30 puntos y test FAB (Frontal Assessment Batery) de 13 puntos. Se realizó una resonancia magnética (RM) de cerebro que reveló múltiples lesiones focales hipertensas e hipercaptantes de predominio supratentorial y distribución corticopial en sustancia blanca subcortical profunda, periventricular y en núcleos grises; lesiones de aspecto nodular, realce lineal perivascular y alzas de realce leptomeningeo en relación a las lesiones superficiales (Figura 2). Cabe destacar la existencia de captación de contaste en ambos pabellones auriculares (Figura 2D), sin evidencia de restricción a la difusión. El electroencefalograma mostró actividad lenta bilateral, sin actividad epiléptica. El estudio de líquido cefalorraquídeo (LCR) fue concordante con meningitis linfocítica de líquido claro (proteínas $0,96 \mathrm{~g} / \mathrm{L}$, glucosa 0,51 g/L, 85 leucocitos con 95\% MN). Los cultivos, estudio TBC, VDRL, panel infeccioso, panel para encefalitis autoinmune y citometría de flujo del LCR resultaron negativos para infección o linfoma. Pruebas sanguíneas infecciosas, y búsqueda de paraproteinemias resultaron negativas. Laboratorio de autoinmunidad evidenció anticuerpos ANA (+) $1 / 80$ Hom, con ENA, DNA, ANCA y TPO negativos y $\mathrm{C} 3$ y C4 fueron normales. Tomografía computada (TC) de tórax, abdomen y pelvis sin hallazgos patológicos.

Es evaluada por reumatología planteándose el diagnóstico de PR (criterios de McAdam y Damini-Levine) con compromiso del SNC. Se realizó una nasolaringofibroscopía que resultó normal. La biopsia de cartílago auricular evidenció pericondritis linfocitaria focal. Posterior a la biopsia auricular se inició tratamiento con metilprednisolona $1 \mathrm{~g} /$ día iv durante 3 días, continuando

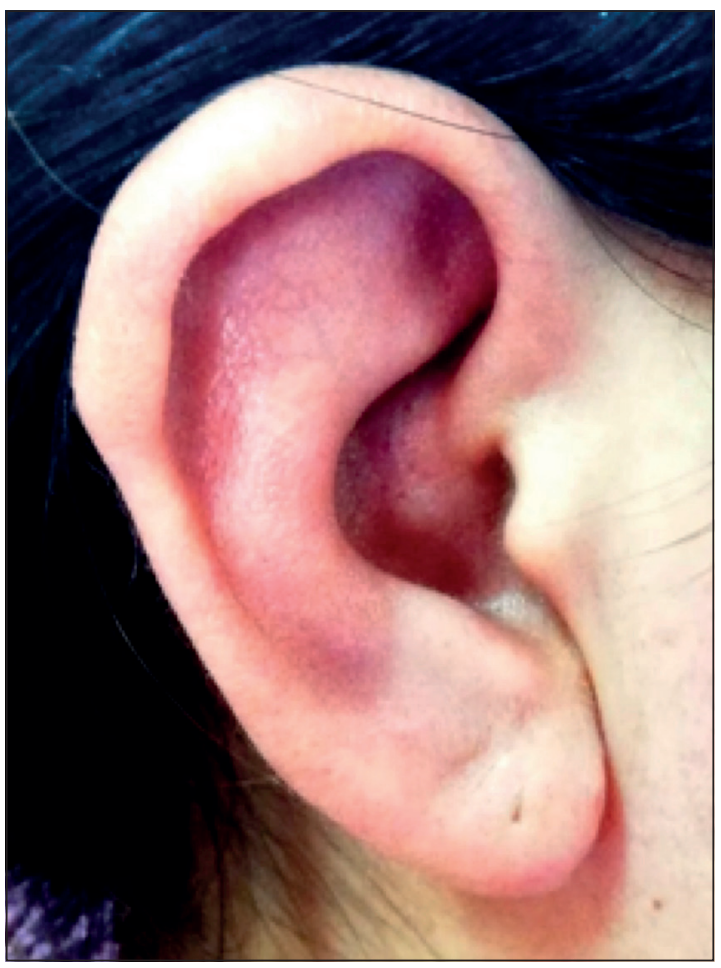

Figura 1. Condritis auricular aguda en una paciente con policondritis recidivante. Se observan signos inflamatorios en el pabellón auricular, mientras que el lóbulo de la oreja se encuentra respetado (estructura que no contiene cartílago). 


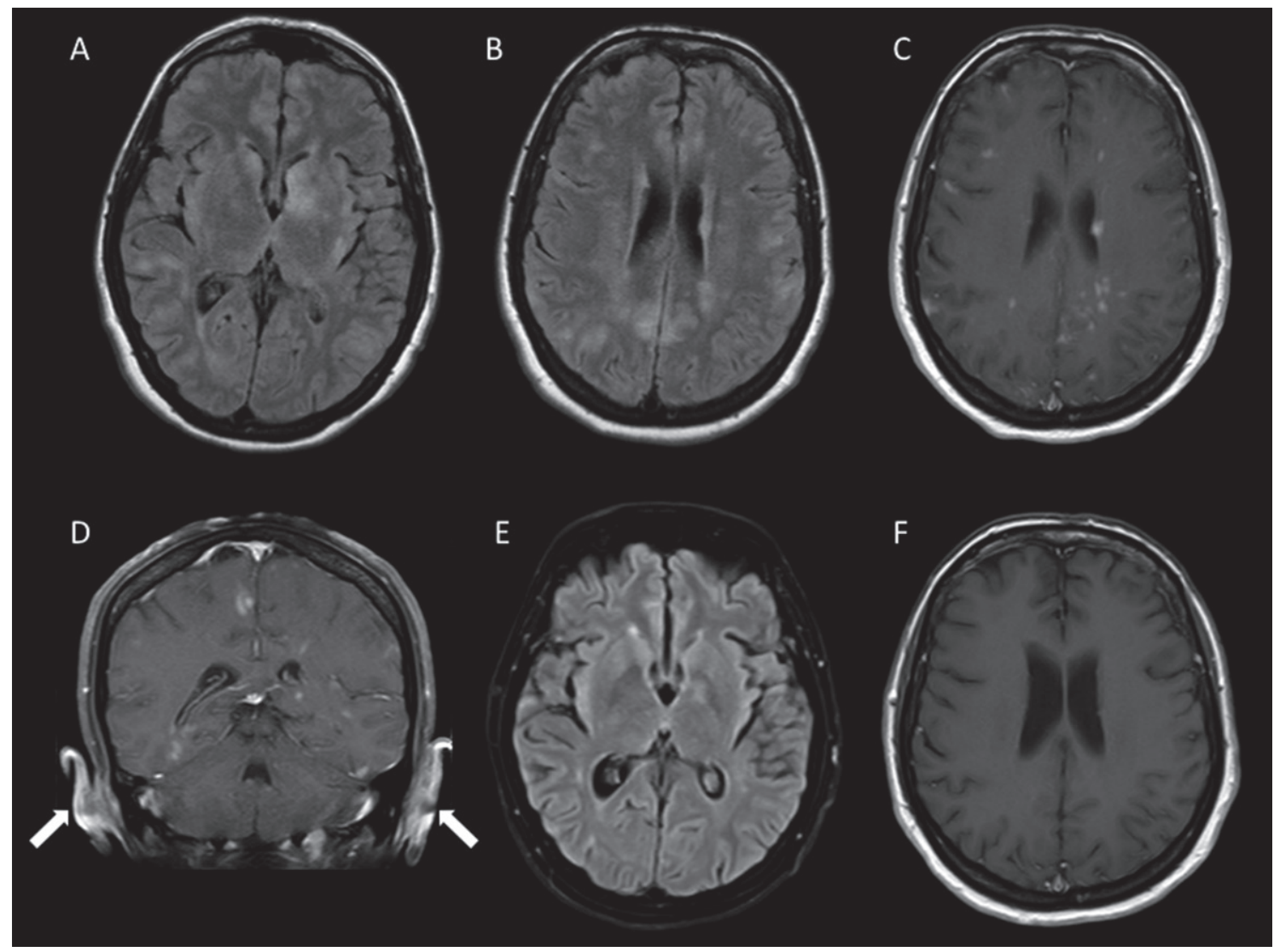

Figura 2. Resonancia magnética de cerebro de paciente con encefalopolicontritis. Múltiples lesiones focales hiperintensas supratentoriales, asimétricas, cortico-subcorticales, en sustancia blanca subcortical, profunda y periventricular y en núcleos grises profundos ( $\mathbf{A}$ y $\mathbf{B}$ ). Varias de ellas presentan realce tras la administración del medio de contraste (C y D), así como el pabellón auricular en ambos lados (ver flechas en $\mathbf{D}$ ). Significativa regresión en el número de lesiones (E) y ausencia de lesiones hipercaptantes (F) en RM cerebral de control a los 2 meses. A y B, FLAIR axial; C, T1 gadolinio axial; D, T1 gadolinio coronal; E FLAIR axial; T1 gadolinio axial.

con prednisona $1 \mathrm{mg} / \mathrm{kg} /$ día por cuatro semanas y posterior reducción gradual de la dosis y ciclofosfamida $100 \mathrm{mg}$ al día. La paciente evolucionó favorablemente con franca mejoría del cuadro neurológico. La RM cerebral de control a los dos meses de tratamiento reveló una significativa regresión de las lesiones (Figura 2 E y F). Al año de evolución la paciente se encuentra asintomática. Paciente autoriza publicación de su caso.

\section{Discusión}

Se presenta el caso de una paciente mujer con un cuadro de encefalopatía subaguda progresiva secundaria a una PR. En nuestro caso la paciente cumple con los criterios de Damiani y Levine: (i) condritis auricular y (ii) confirmación histológica de condritis ${ }^{2}$. Se descartó la existencia de un lupus eritematoso sistémico.

Las manifestaciones neurológicas asociadas a la PR son diversas, siendo la más frecuente la neuropatía craneal del VIII, VII, VI y II ${ }^{3}$. Se han reportado hemiplejia, ataxia, mielitis y polineuropatía. Más raramente, pueden desarrollarse meningitis aséptica, meningoencefalitis, encefalitis límbica, accidente cerebrovascular, convulsiones focales o generalizadas y aneurismas cerebrales ${ }^{4,5}$.

Los hallazgos radiológicos son diversos y dependen del síndrome neurológico presentado por 
el paciente. En la TC de cerebro se han reportado engrosamientos de senos paranasales, tejidos blandos periorbitarios y pabellones auriculares. Por otra parte, la RM cerebral puede ser normal, mostrar algún grado de atrofia u otras alteraciones dependiendo la evolución y grado de la enfermedad, incluyendo el "signo de la oreja prominente" en la secuencia de difusión ${ }^{6}$, el cual no estaba presente en nuestra paciente. De forma más o menos consistente se han descrito lesiones hipertensas multifocales en T2 y FLAIR, de ubicación cortical, sustancia blanca y en ganglios de la base, las cuales suelen ser hipercaptantes ${ }^{6}$.

La fisiopatología de la encefalopolicrondritis aún es desconocida, sin embargo, algunos autores la han atribuido a mecanismos meningoencefalíticos o vasculíticos propios de la $\mathrm{PR}^{7,8}$. Tras realizar la autopsia de un paciente con PR con representación de demencia por cuerpos de Lewy se propuso la participación de la inmunidad humoral, con anticuerpos contra colágeno I, IX, X y matrilina-1 (proteínas de los vasos cerebrales), así como la formación de complejos antígeno-anticuerpo con activación del complemento desencadenando un proceso inflamatorio en la vasculatura cerebral. Otro mecanismo patogénico propuesto es la activación de células T que infiltran el sistema nervioso central debido a una respuesta inflamatoria no mediada por anticuerpos. En los escasos estudios histopatológicos se ha reportado infiltración linfocítica, acumulación de depósitos granulares de inmunoglobulinas. Por otra parte, en 2 casos de encefalitis límbica no herpética asociados con policondritis recurrente, las biopsias cerebrales revelaron gliosis marcada, manguitos perivasculares con engrosamiento y destrucción de la pared vascular en vasos meníngeos e intracerebrales ${ }^{9}$.

Con adecuado tratamiento, la sobrevida de los pacientes con PR es de $74 \%$ a 5 años ${ }^{10}$, siendo la neumonía la principal causa de muerte, debido a la estenosis progresiva de las estructuras de la vía aérea superior. Otras causas de muerte son las complicaciones renales, cardiovasculares y neurológicas. La mortalidad de la PR asociada a meningitis alcanza $12 \%$, aumentando a $36 \%$ en el caso de encefalitis ${ }^{11}$. El reconocimiento temprano de la afectación del SNC en la PR es extremadamente importante, debido a que la rápida administración de corticoesteroides e inmunosupresores tienen el potencial de modificar la evolución y pronóstico de la enfermedad ${ }^{12}$. Algunos casos han sido tratados con esteroides en altas dosis durante la fase de exacerbación o recidiva y la administración de inmunoglobulina endovenosa ha sido reportada recientemente con éxito en la remisión de la enfermedad ${ }^{13}$. Respecto al tratamiento a largo plazo éste incluye el uso de dapsona, azatioprina, ciclofosfamida, metotrexato $\mathrm{y}$ anticuerpos monoclonales.

Aunque la respuesta al tratamiento precoz con corticoides es importante, algunos casos de PR con encefalitis límbica continúan con alteraciones de memoria posterior al tratamiento. En estos casos, las imágenes de RM muestran atrofia del lóbulo temporal medial y dilatación del asta temporal del ventrículo lateral, proponiéndose como causa un daño isquémico irreversible. Además, al realizar SPECT del cerebro de estos pacientes, se evidenció una menor irrigación de región temporomesial con afección bilateral ${ }^{14}$.

Finalmente, aunque se trata de una enfermedad muy poco frecuente y no descrita antes en nuestro país, creemos que es importante tenerla en cuenta en el diagnóstico diferencial de pacientes con cuadros de encefalopatía asociada a alteraciones inflamatorias mucocutáneas, cartilaginosas y articulares, para iniciar rápidamente el tratamiento esteroidal e inmunosupresor adecuado.

\section{Referencias}

1. McAdam LP, O'Hanlan MA, Bluestone R, Pearson CM. Relapsing poly- chondritis: prospective study of 23 patients and a review of the literature. Medicine (Baltimore) 1976; 55: 193-215.

2. Damiani JM, Levine HL. Relapsing polychondritis. Report of ten cases. Laryngoscope 1979; 89: 929-43.

3. Irani SR, Soni A, Beynon H, Athwal BS. Relapsing "encephalo" polychondritis. Practical Neurology 2006; 6: 372-75.

4. Sampaio L, Silva L, Mariz E, Ventura F. Central nervous system involvement in relapsing polychondritis. Joint Bone Spine 2010; 77 (6): 619-20.

5. ZJ Wang, CQ Pu, ZJ Wang, JT Zhang, XQ Wang, SY Yu, et al. Meningoencephalitis or Meningitis in Relapsing Polychondritis: Four Case Reports and a Literature Review. J Clin Neurosci. 2011 Dec; 18 (12): 1608-15.

6. Kuwabara M, Shimono T, Toyomasu M, Shioyama M, Mitsui Y, Yoshinaga E, et al. "Prominent ear sign" on diffusion-weighted magnetic resonance imaging in 
relapsing polychondritis. Radiation Medicine 2008; 26 (7): 438-41.

7. Head E, A Starr, RC Kim, A Parachikova, GE Lopez, M Dick, et al. Relapsing Polychondritis With Features of Dementia With Lewy Bodies. Acta Neuropathol 2006; 112 (2): 217-25.

8. Stewart SS, Ashizawa T, Dudley AW, Goldberg JW, Lidsky MD. Cerebral vasculitis in relapsing polychondritis. Neurology 1988; 38 (1): 150-2.

9. Ohta Y, Nagano I, Niiya D, Fujioka H, Kishimoto T, Shoji M, et al. Nonparaneoplastic limbic encephalitis with relapsing polychondritis. Journal of the Neurological Sciences 2004; 220 (1-2): 85-8.

10. Michet CJ Jr, McKenna CH, Luthra HS, O’Fallon WM. Relapsing polychondritis: Survival and predictive role of early disease manifestations. Ann Intern Med. 1986; 104
(1): 74-8.

11. Jeon CH. Relapsing Polychondritis with Central Nervous System Involvement: Experience of Three Different Cases in a Single Center. J Korean Med Sci. 2016; 31 (11): 1846-50.

12. Ellis RJB, Mbizvo GK, Jacob A, Doran M, Larner AJ. Relapsing polychondritis complicated by cognitive dysfunction: two distinct clinical phenotypes? International Journal of Neuroscience 2016; 127 (2): 124-34.

13. Terrier B, Aouba A, Bienvenu B, Bloch-Queyrat C, Delair E, Mallet J, et al. Complete remission in refractory relapsing polychondritis with intravenous immunoglobulins. Clin Exp Rheumatol. 2008; 26: 136-8.

14. Fujiki F. Non-herpetic limbic encephalitis associated with relapsing polychondritis. J Neurol Neurosurg Psychiatry 2004; 75 (11): 1646-7. 